\title{
CONTROL OF OSCILLATION PATTERNS IN A SYMMETRIC COUPLED BIOLOGICAL OSCILLATOR SYSTEM
}

\author{
Atsuko Takamatsu ${ }^{*+1}$, Reiko Tanaka ${ }^{\S}$, Takatoki Yamamoto ${ }^{\dagger}$, and \\ Teruo Fujii ${ }^{\dagger}$ \\ *PRESTO, Japan Science and Technology Corporation (IST), Japan \\ ${ }^{\dagger}$ Underwater Technology Research Center, Institute of Industrial Science, The University of Tokyo, \\ 4-6-1, Komaba, Meguro-ku, Tokyo, 153-8505, Japan \\ 'Dept. of Applied Physics and Physico-Informatics, Keio University, Hiyoshi, Kohoku-ku, Yokohama, \\ 223-8522, Japan \\ ${ }^{\S}$ Control and Dynamical Systems, California Institute of Technology, MC 107-81 \\ Pasadena, CA, 91125, USA
}

\begin{abstract}
A chain of three-oscillator system was constructed with living biological oscillators of phasmodial slime mold, Physarum polycehalum and the oscillation patterns were analyzed by the symmetric Hopf bifurcation theory using group theory. Multi-stability of oscillation patterns was observed, even when the coupling strength was fixed. This suggests that the coupling strength is not an effective parameter to obtain a desired oscillation pattern among the multiple patterns. Here we propose a method to control oscillation patterns using resonance to external stimulus and demonstrate pattern switching induced by frequency resonance given to only one of oscillators in the system.
\end{abstract}

\section{INTRODUCTION}

Most of biological systems can be modeled with geometrical symmetries. For example, neuronal systems for control of animal locomotion consist of central nervous system and ganglions arranged symmetrically. Such systems could be modeled by a coupled oscillator system that has potential to show a variety of oscillation patterns, namely, a variety of locomotion patterns. One of the most popular parameters to control the patterns would be coupling strength between oscillators. It is, however, ineffective to obtain a desired pattern among multiple patterns if the system has multistability. Here we propose a method to control the oscillation patterns by controlling the frequencies of a single oscillator in symmetric coupled oscillators.

We constructed three-oscillator system in a chain with living biological oscillators of plasmodial slime mold, Physarum polycehalum. The plasmodial slime mold is an amoeboid multinucleated unicellular organism and shows various oscillatory phenomena in the concentration of intracellular chemicals such as ATP and $\mathrm{Ca}^{2+}$ that are considered to lead contraction/relaxation states in the cell, namely, thickness

${ }^{1}$ Electronic address: atsuko@iis. u-tokyo.ac.jp

CP676, Experimental Chaos: $7^{\text {th }}$ Experimental Chaos Conference,

edited by V. In, L. Kocarev, T. L. Carroll, B. J. Gluckman, S. Boccaletti, and J. Kurths (c) 2003 American Institute of Physics 0-7354-0145-4/03/\$20.00 
oscillation of the cell, induced by complicated mechanochemical reactions among the chemicals, proteins, and intracellular organelles. The thickness oscillation causes protoplasmic streaming inside tube structures of the plasmodial cell, which would relate to interactions between partial bodies of the cell. Thus, this system can be regarded as an assemble of nonlinear oscillators coupled by the tube structure. We have constructed two-oscillator systems and three-, four-, five-oscillator systems in rings by a cell patterning method and have shown that the symmetrically arranged oscillator-systems exhibit multiple oscillation patterns predicted by the symmetric Hopf bifurcation theory using geometrical symmetry proposed by Golubitsky and Stewart [1-4]. In order to obtain more information on the system, we investigated the responses to environmental change. As one of the results, we demonstrate pattern switching between different oscillation patterns induced by frequency resonance in order to control the multi-stable patterns.

\section{METHODS}

A three-oscillator system in a chain was constructed with a microfabricated structure of SU-8 (photoresist resin) as shown in Fig. 1(a),(b) by a method previously reported $[1,3]$. The structure consists of oscillator parts, where thickness oscillation is observed, and channel structures. The coupling strength between the oscillators can be controlled with the channel width in the microfabricated structure [1]. In this experiment, the channel width was fixed at $400 \mu \mathrm{m}$ that corresponds to strong coupling. In this condition, the system could show multiple oscillation patterns [2]. It is known that oscillation frequency in the plasmodium is affected by temperature change [5]. A chip for temperature control was fabricated by patterning a transparent resistant material, ITO (Indium Tin Oxide), on a glass substrate to be used as multi-channel sensors and heaters. Local stimuli in small areas $(2 \times 2 \mathrm{~mm})$ can be applicd to multiple points with small distances $(4 \mathrm{~mm})[6,7]$. The plasmodial oscillator system was placed on this chip. Moreover, transparency of ITO material is useful for optical observation of cells. Stimuli were applied by periodic change of temperature to one of the oscillators located in the center (Oscillator 2; denoted as $\mathrm{O} 2$ in Fig. 1 (c)). Temperature of the other oscillators was maintained at $21^{\circ} \mathrm{C}$.

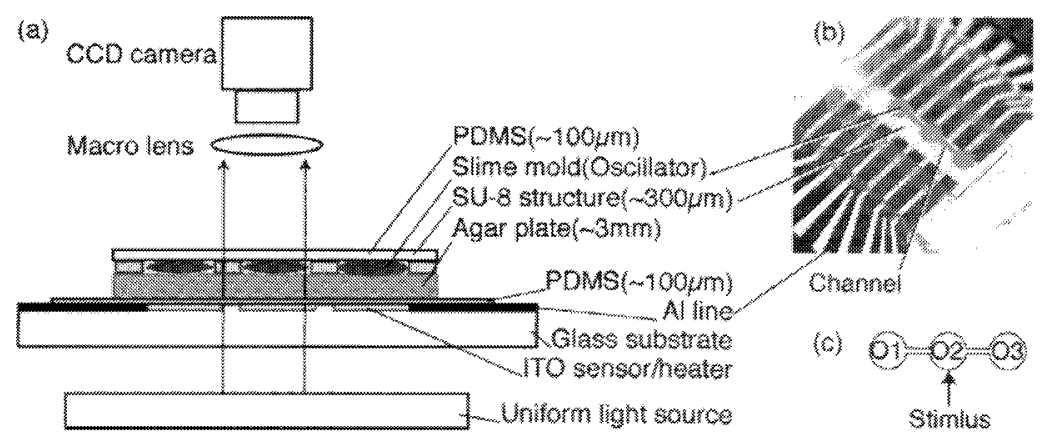

FIGURE 1. (a), (b) Experimental setup. (c) Three-oscillator system in a chain. 


\section{OSCILLATION PATTERNS}

The system shows multiple oscillation patterns irrespective of existence of stimulus by temperature change. In Fig.2 (a), oscillator 1 (O1) and $3(\mathrm{O} 3)$ show anti-phase oscillation while oscillator $2(\mathrm{O} 2)$ shows double frequency compared with the other oscillators (pattern A). In Fig.2 (b), adjacent oscillators are in anti-phase (pattern B). In Fig.2 (c), the frequencies of all oscillators are doubled (pattern C). As seen in the time series of thickness oscillation in Fig. 2 (c), the frequencies are original before the time $200(\mathrm{sec})$, and doubled after the time $200(\mathrm{sec})$ (denoted with a gray box). In Fig.2 (d), the phases in adjacent oscillators are shifted by $90^{\circ}$ then the phase wave propagates from one end to another end (pattern D). The observed patterns were dependent on the sample of the plasmodium even when the coupling strength was fixed, which implies that this system has multi-stability. In addition, spontaneous pattern switching was observed except between the pattern $\mathrm{A}$ and $\mathrm{B}$. The most frequently observed pattern was A $(41 \%$; in number of periods in 30 minutes observation of 33 samples), secondly C (13\%) and D (12\%), followed by B (7\%), when the coupling strength is fixed at one parameter. The probability of the observed patterns depends on the coupling strength.

By the group theory, this system has $Z_{2}$-symmetry that has a reflection denoted with $\beta$ in Fig. 2 (e). The symmetric Hopf bifurcation predicts the patterns $A$ and in the system with $Z_{2}$-symmetry [4]. The pattern $A$ keeps spatio-temporal symmetry under the action of reflection $\beta$ with a phase shift of $180^{\circ}$. Double frequency of $\mathrm{O} 2$ in the pattern $\mathrm{A}$ is generated to keep the symmetry when the phase is shifted by $180^{\circ}$, since $\mathrm{O} 2$ is located on the reflection axis. The pattern $\mathrm{B}$ keeps the symmetry under only the reflection $\beta$. The pattern $\mathrm{D}$, however, dose not keep the symmetry under the reflection $\beta$ with any phase shift. This oscillation pattern would be generated by $D_{4}$-symmetry where a hidden oscillator is assumed, whose details will be reported elsewhere. Double frequency of all oscillators in the pattern $\mathrm{C}$ cannot be explained by $Z_{2^{-}}$ symmetry, which could be caused by system specific characteristics of the plasmodium. For simplicity, we focus on only the patterns A and B in this report and, in order to control the patterns $\mathrm{A}$ and $\mathrm{B}$, we applied the external stimulus to the center oscillator to keep $Z_{2}$-symmetry.

\section{PATTERN SWITCHING BY RESONANCE}

We applied the stimulus by change of temperature to the center oscillator $\mathrm{O} 2$ and changed the only one control parameter $\omega_{f} / \omega_{0}$ from 0.5 to 2.0 , where $\omega_{f}$ is an angular frequency of stimulus and $\omega_{0}$ is an original angular frequency of 01 and 03 before stimulus $\left(\omega_{0} \equiv \omega_{1} \sim \omega_{3}, \omega_{0} \sim \omega_{2} / 2\right.$ in the pattern $A, \omega_{0} \sim \omega_{1} \sim \omega_{2} \sim \omega_{3}$ in the pattern $\left.B\right)$.

Fig. 3 shows an example of response to the periodic stimulus. Temperature was changed in a sinusoidal function form $\left(T_{0}+A \sin \left(\omega_{f} t+\phi\right) ; T_{0}=21.0^{\circ} \mathrm{C}, A=1.5^{\circ} \mathrm{C}, \phi\right.$ is an arbitrary initial phase) from the time around 1900 (sec) as shown in Fig. 3(a). The system showed the oscillation pattern A before starting the stimulus and the pattern B 
after several periods of stimulation. When the stimulus started, the double frequency of $\mathrm{O} 2$ was destroyed, then the dynamical change in phase difference between $\mathrm{O} 1$ and O3 from $180^{\circ}$ to $0^{\circ}$ was observed at the time around 3500 (sec) in Fig. 3 (f). Switching from the pattern $\mathrm{B}$ to $\mathrm{A}$ was also observed under different condition of stimulus (data not shown).

The mechanism of the switching is considered as follows. Each plasmodial oscillator shows resonance against external periodic stimulus which means the oscillator synchronize with external the stimulus to change its frequency.

(a)
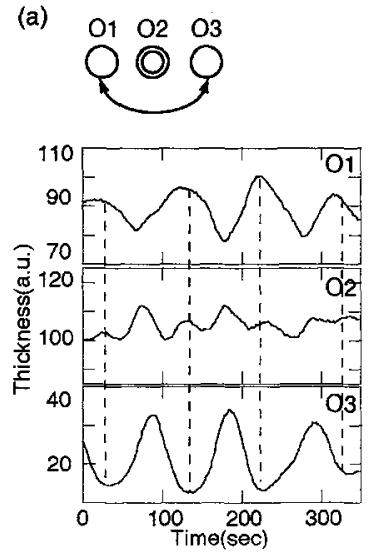

(c)
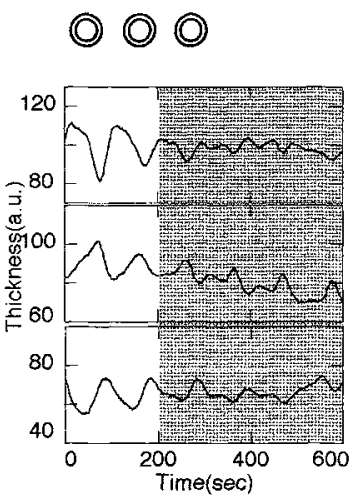

(61) (0) (b)

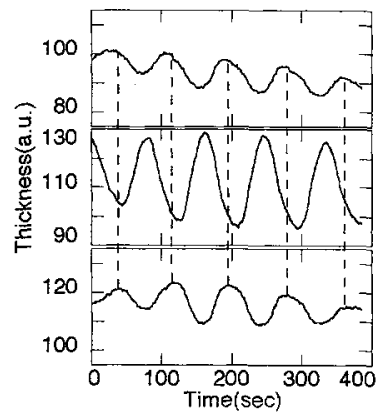

(d)
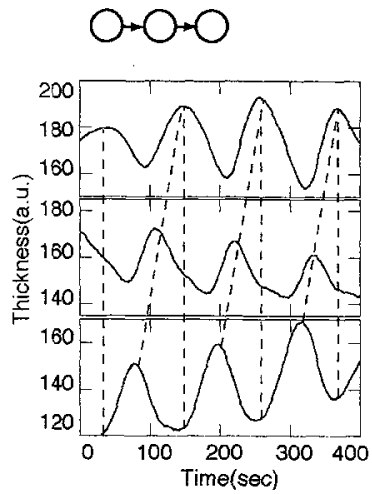

FIGURE 2. (a-d) Schematic diagrams of oscillation patterns and thickness oscillation. Open circles are oscillators. Concentric circles are oscillators have double frequency. Bidirectional and unidirectional arrows mean that phase difference between oscillators is $180^{\circ}$ and $90^{\circ}$, respectively. (e) $Z_{2}$ symmetry in a chain of three oscillators. $\beta$ is a reflection. 


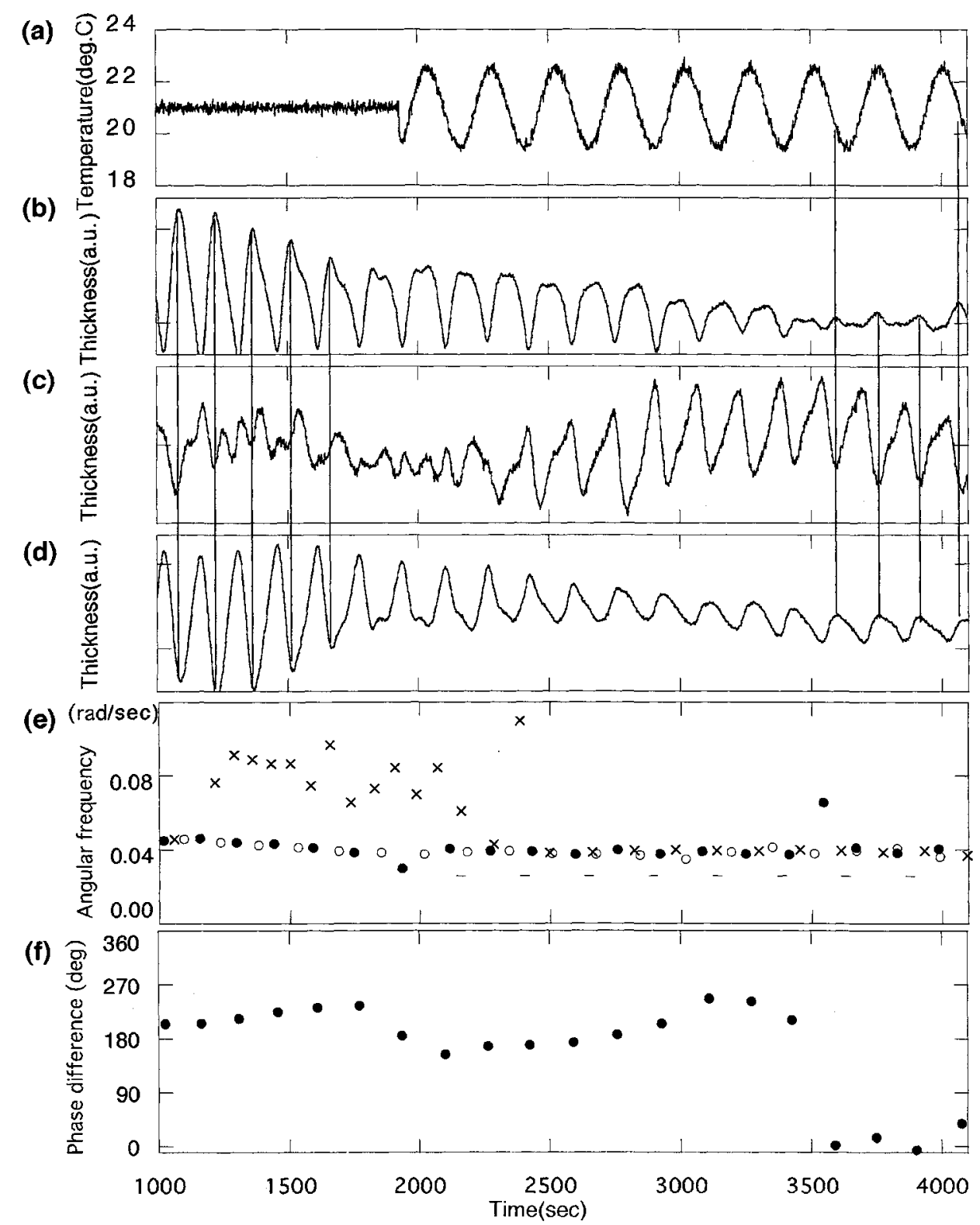

FIGURE 3. Pattern switching by periodic force. (a) Stimulus by temperature change. (b-d) Thickness oscillation in oscillator 1-3. (e) Angular frequency. Bars, filled circles, crosses, open circles are those of stimulus, thickness change in Oscillator 1,2 and 3, respectively. Original frequencies: $\omega_{1}=0.044 \pm 0.003$, $\omega_{2}=0.083 \pm 0.010, \omega_{3}=0.043 \pm 0.002$. Frequency of the stimulus: $\omega_{f}=0.026$. Frequencies during stimulus: $\omega_{1}=0.040 \pm 0.001, \omega_{2}=0.040 \pm 0.001, \omega_{3}=0.039 \pm 0.002$. (f) Phase difference of oscillation between oscillator 1 and 3 . 
In one oscillator system of the plasmodium, we have observed 1:1, 1:2, 1:3 $\left(\omega: \omega_{;} ; \omega\right.$ is angular frequency of $\mathrm{O} 1$ or $\mathrm{O} 3$ during stimulus) resonances and the resonances in other fractional number combination such as 3:2, 4:5, etc., and have found that its phase diagram has Arnol'd tongues [8]. If $\omega_{f} / \omega_{0}$ is within the tongue, $\mathrm{O} 2$ resonates with a different frequency $\omega$ from original $\omega_{0}$. In the case of Fig. 3, $\omega_{0} \sim 0.044 \pm 0.003$ and $\omega \sim 0.039 \pm 0.002$ and the system shows 3:2 resonance. It should be noted that the frequency was reduced to $86 \pm 9(\%)$ of the original in all the samples where switching from the patterns A to B was observed. The system showed the resonance in an appropriate ratio in most cases. If the stimulus was applied to increase the frequency, pattern switching occurs from A to B was not observed. On the other hand, the frequency increased when the switching from B to A. Consequently, it is concluded that the control between the pattern A and B can be achieved only by controlling the frequency of the center oscillator that affects on both ends of oscillators symmetrically.

\section{CONCLUSION}

We demonstrated the control of the oscillation patterns by using the resonance to external stimulus in the multi-stable system. The multi-stability of the system is commonly observed in other systems of coupled oscillators as well [9]. Thus, the method we demonstrated here could be generally effective to control such system if the oscillation pattern depends on the frequency. Furthermore, we observed the dynamical pattern switching from/to in-phase to/from anti-phase by taking notice of the oscillators in both ends. The anti-phase mode, for example, corresponds to walking mode in bipedal animal and in-phase mode corresponds to hopping mode. This method might also be useful as the model of the control of animal locomotion. Moreover, it should be stated the importance of consideration of the symmetry in the system which generally observed in nature $[4,10]$.

\section{REFERENCES}

1. Takamatsu, A. , Fujii, T., Endo, I. Phys. Rev. Lett. 852026 (2000).

2. Takamatsu, A. et al., Phys. Rev. Lett. 87078102 (2001).

3. Takamatsu, A. and Fujii, T. "Construction of a living coupled oscillator system of plasmodial slime mold by a microfabricated structure" in Sensors Update Vol. 10, edited by Wiley-VCH, Weinheim, 2002, pp. 33-46.

4. Golubitsky, M. and Stewart, I., The Symmetry perspective, Birkhäuser Verlag, Basel, 2001.

5. Matsumoto, K. et al. J. Theor. Biol, 131175 (1988).

6. Yamamoto, T. Nojima, T. and Fujii, T. "PDMS-glass hybrid microreactor for cell-free protein analysis" (2002) submitted.

7. Takamatsu, A. and Fujii, T., "Observation of response to stimuli in oscillating cells patterned by microfabricated structure" Proceedings of The Sixth International Symposium on Micro Total Analysis System ( $\mu T A S s)$, Nara, Japan, 2002, to appear.

8. Takamatsu, A., Yamamoto, T. and Fujii, T., "Resonant pattern in a plasmodial slime mold by periodic force of temperature." (2002) in preparation.

9. Yoshimoto, M, Yoshikawa, K. and Mori, Y. Phys. Rev. E 47864 (1993)

10. Tanaka, R., Iwata, S and Shin, S. Trans. of the Society of Instrument and Control Engineers 33441 (1997). 